\title{
Yellow Supergiants and R Coronae Borealis Stars
}

\author{
P.L. Cottrell \\ Mount John University Observatory, Dept of Physics $\mathscr{B}$ Astronomy, \\ University of Canterbury, Christchurch, New Zealand
}

\begin{abstract}
The study of luminous variable stars has important consequences both with respect to the scale of the Universe and for our understanding of the later stages of stellar evolution.

This review will look at a number of groups of these stars, in particular the RV Tauri stars, the hydrogen-deficient Carbon (HdC) stars, including the R Coronae Borealis (RCB) stars and some aspects of Cepheid variables.

Both photometric and spectroscopic observations will be presented to show the amount of progress that has been made in this area over the last several years. Links will be made to various models which are being used to understand the observational data.

The most important conclusion is that there is still enormous scope for the acquisition and detailed analysis of data on the relatively bright members of these groups of stars. These can be used to provide further understanding of the more distant objects of each group and of the wider problems in astrophysics.
\end{abstract}

\section{Introduction}

This paper will investigate a subset of objects presented in John Percy's 1993 paper, "Supergiant variables: recent observational results". I will confine myself to the yellow supergiant variables of low mass, as well as some aspects of Cepheid variables.

The talk will reinforce one of Percy's comments. The study of non-linear processes in supergiant variables requires long series of observations of good and consistent quality. In addition, it is also appropriate to add Percy's next sentence. Long-term projects are often not encouraged or supported in astronomy. They are sometimes regarded as being simply 'stamp-collecting', a term Ernest Rutherford used to describe all aspects of science apart from physics.

To gain recognition for this type of research it is imperative that the focus must be to provide observational tests of existing models or to prompt specific developments in this modelling. Although not exclusively, this has generally been confined to stellar envelope models, but with the acquisition of high quality spectral data, there is a need to develop appropriate dynamical model atmospheres to interpret the observational data.

The late stages of stellar evolution provide objects which can be used for a multitude of astrophysical purposes. Several examples of these pieces of work 


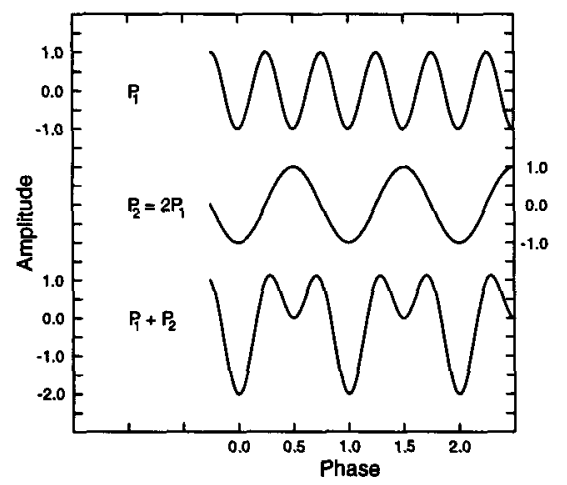

Figure 1. A schematic representation of the alternating behaviour of RV Tauri stars (bottom), using two precisely phased sinusoids of periods $P_{1}$ and $P_{2}$.

are: studies of stellar pulsation; tests of stellar evolutionary theory; investigation of circumstellar shells; determination of stellar masses; evolutionary links between high luminosity objects (e.g., W Vir to RV Tau to SRd and RCB to planetary nebula to white dwarf); determination of the distance scale; understanding the precision of this scale.

There is also scope for investigating other types of connections, in addition to evolutionary ones, as they can provide insights into the physical processes which are occurring. Two examples of this type of analogy are Shock waves in stellar atmospheres and breaking waves on an ocean beach by Wallerstein \& Elgar (1992) and Of stars \& trumpets by Sasselov (1993).

\section{Observational program}

As an example of the type of long-term observational program that is required to address some of the issues mentioned above, I will discuss the program that we have developed at Canterbury. I will combine it with other researchers' programs to complement the discussion.

The Mount John University Observatory (MJUO) program has involved a symbiotic relation between the facilities available and the types of astrophysical programs which we wanted to undertake. It follows some of the ideas of Warner (1986) by using well-equipped and well-calibrated instruments on small telescopes, gaining regular access to telescope time, employing resident observers and doing appropriate research. This has lead to a number of long-term photometric and spectroscopic studies of regular and semi-regular variable stars.

The specific types of objects that I will consider are RV Tauri stars, HdC stars, with particular attention to the RCB stars, and Cepheid variables. In most of these studies we combine the photometric and spectroscopic data, the latter in the form of line profile variations, stellar radial velocities and abundance 


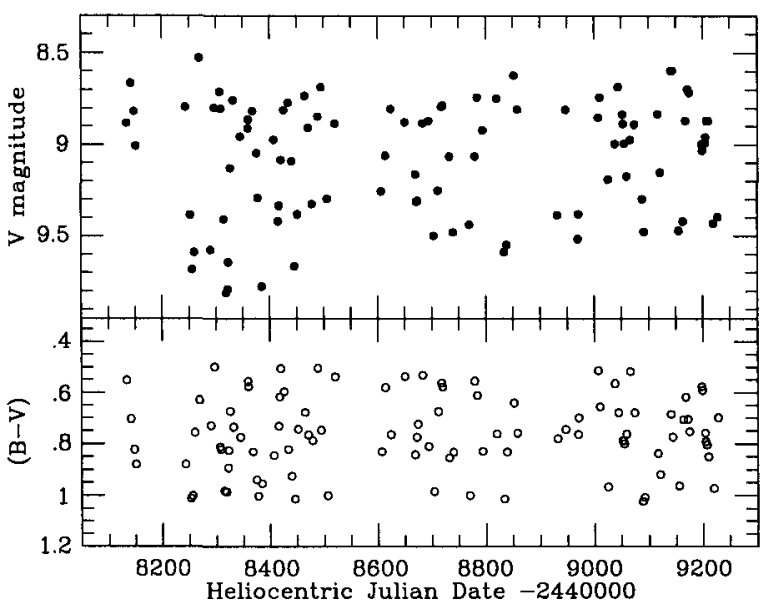

Figure 2. MJUO $V$ and $(B-V)$ photometry of the RVa star, RU Cen.

determinations, to understand the evolutionary and dynamical properties of these systems.

\section{RV Tauri stars}

Much of the new work described in this section is the PhD thesis work of Karen Pollard. It exemplifies the discussion in the previous section and presents some significant new work.

\subsection{Previous work}

It seems appropriate to begin my overview using Wahlgren (1992) as the starting point. This paper discussed the class of stars in significant detail, in particular the alternating photometric behaviour (which is the principle defining characteristic of these stars, see Figure 1), their infrared excesses and the general spectroscopic properties of the systems (see also Preston et al. 1963).

There is a general consensus that the RV Tauri stars are low mass objects in transition from AGB to white dwarf phase. The infrared excess is indicative of a relatively recent phase of mass loss which has contributed towards an extensive circumstellar envelope. The more recent spectroscopic work (Lèbre \& Gillet 1991a,b; Gillet 1992) has included higher resolution than the earlier work and has enabled more precise radial velocities to be obtained and investigation of atmospheric stratification effects and the propagation of shock waves through the photospheric layers.

Before discussing the new data it is appropriate to define the major divisions in this class of star. Photometrically, they have been divided into two sub-classes as a consequence of their light amplitude. The RVa sub-class are stars which, although showing the alternating behaviour, tend to have a maximum brightness which is constant, whereas the RVb sub-class have a maximum 


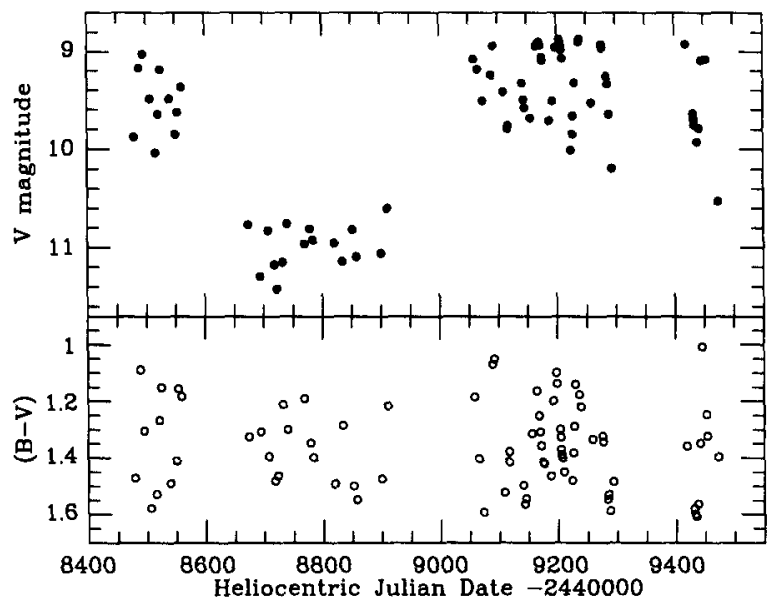

Figure 3. MJUO $V$ and $(B-V)$ photometry of the RVb star, AI Sco.

brightness which changes over timescales of 500 to 2000 days. Spectroscopically, Preston et al. (1963) defined a number of classes as well, using ' $A$ ', ' $B$ ' and ' $C$ ' as the designators, respectively corresponding to G-K type variables and Fp type with enhanced and weak molecular carbon features.

One important paper which relates well to the type of study which I outlined in the general introduction is the work by Shenton et al. (1992), which used contemporaneous IUE, optical and infrared data to model a substantial fraction of the electromagnetic spectrum of AC Her.

There have been a number of spectroscopic studies which have determined photospheric compositions for one or more of these stars (see e.g., Luck \& Bond 1984, 1989; Giridhar et al. 1994). Most studies have shown that these stars have varying degrees of metal deficiency and with some evidence of carbon enhancement in type ' $\mathrm{B}$ ' objects.

\subsection{MJUO program}

A photometric BVRI program has been undertaken on 11 stars and has extended over about 4 years. Figures 2 and 3 show examples of the photometry for RU Cen (RVa) and AI Sco (RVb). It has been supported by contemporaneous spectroscopy at MJUO (and supplemented by some spectra obtained on the Mount Stromlo Observatory 1.9-m telescope). These spectra enabled the investigation of the phase dependent behaviour of $\mathrm{H} \alpha$ and some of the metallic lines and the general systematic trend in velocity as a function of phase to be determined.

To enable a detailed comparison of the class of stars as a whole, all these photometric data have been phased (using the spacing between successive deep minima as the defining period). (For the RVb sub-class the long-term trends in the data were removed prior to phasing.) This has shown that there are significant differences between individual stars and also between different cycles of the same star. 


\subsection{Modelling}

There are two main models which have been developed to describe the alternating behaviour of the RV Tauri stars. These are the resonance hypothesis (see Takeuti \& Petersen 1983; Tuchman et al. 1993; Fokin 1994) and a period doubling processes (Buchler \& Kovács 1987; Aikawa 1993; Buchler et al. 1995), which these latter authors use to describe the RV Tauri stars as being indicative of a transition from regular to irregular (chaotic) pulsations. Certainly the semi-regular nature of the photometric and spectroscopic observations is going to require some individual attention in describing each of these stars. Buchler et al. (1995) also reinforce the idea that continuous (or almost continuous) observations of irregular variable stars could provide testbeds for chaos theory.

Table 1. Orbital parameters for $v$ Sgr and LSS 4300

$\begin{array}{lcccc} & v \text { Sgr (1929) } & v \text { Sgr (1990) } & v \text { Sgr (1992) } & \text { LSS 4300 } \\ P(\mathrm{~d}) & 137.95 & 137.943 \pm 0.004 & 137.89 \pm 0.17 & 52.09 \pm 0.02 \\ \gamma\left(\mathrm{kms}^{-1}\right) & 13.3 & 7.4 \text { (fixed) } & 13.0 \pm 1.3 & -5.1 \pm 4.2 \\ \mathrm{~K}\left(\mathrm{kms}^{-1}\right) & 49.1 \pm 0.8 & 47.4 \pm 0.8 & 46.6 \pm 1.7 & 56.9 \pm 3.4 \\ \mathrm{e} & 0.060 \pm 0.016 & 0 & 0.065 \pm 0.039 & 0.38 \pm 0.06 \\ \omega\left(^{\circ}\right) & 16.8 \pm 9.5 & - & 23 \pm 24 & 47.1 \pm 7.3 \\ \mathrm{~T}^{a} & -4.7 \pm 0.1 & 7916.2 \pm 0.4 & 4889 \pm 16 & 5025.6 \pm 2.0 \\ \operatorname{asin} i(\mathrm{Gm}) & 90.3 & - & 88.21 \pm 3.3 & 37.7 \pm 2.5 \\ \mathrm{~F}(\mathrm{~m}) & 1.677 & 1.52 \pm 0.08 & 1.44 \pm 0.16 & 0.79 \pm 0.15\end{array}$

${ }^{a_{\text {zero }}}$ epoch is JD 2440000

\section{HdC stars}

In this area I will describe two types of semi-regular variables for which our understanding has been significantly enhanced by the type of approach that we have taken. One type is the hydrogen-deficient binaries (HdB) and the other is the RCB stars.

\subsection{HdB}

Some of the new work that I will describe was undertaken by David Frame, a master of science student at Canterbury.

Previous work There has been limited photometry and spectroscopy on this group of stars, particularly as three ( $v$ Sgr, LSS4300 and CPD-58 2721 ) of the four confirmed members are southern objects. Greenstein, between 1940 and 1950 , undertook an extensive spectroscopic study of $v \mathrm{Sgr}$, in which he deduced the hydrogen deficiency of the atmosphere, confirmed the orbital variations of some features and the non-orbital variation of others, particularly $\mathrm{H} \alpha$. Malcolm \& Bell (1986) and Morrison (1988) obtained limited photometric data on a 


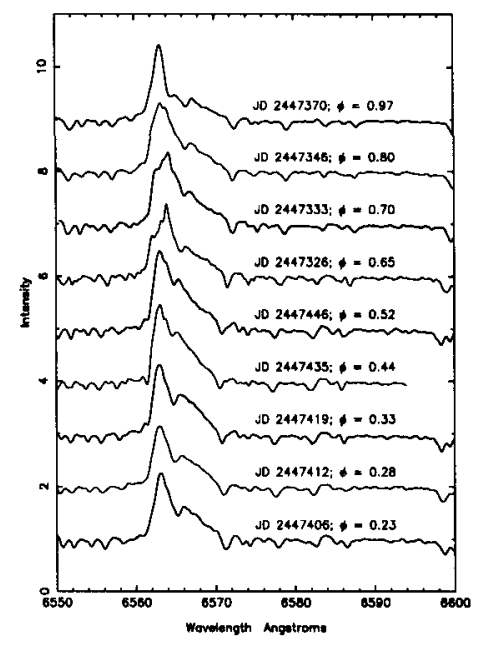

Figure 4. Sample of $v$ Sgr spectra as a function of orbital phase.

number of these stars, but were only able to deduce that there were some shortterm $(\sim 10-20 \mathrm{~d})$ variations.

More recently Dudley \& Jeffrey $(1990,1993)$ have combined various data, including IUE spectra, to recalculate the binary star orbit and through a model atmosphere analysis derived temperatures and luminosities for the primary star (the hydrogen-deficient member) of the system.

MJUO program and modelling We have undertaken long-term photometry of the three southern HdB stars. In addition, spectroscopy was obtained over an extended period for $v$ Sgr and LSS4300 and orbits determined (see Table 1). The $v$ Sgr orbit (labelled 1992) is similar to that obtained by Dudley \& Jeffrey (1990), labelled 1990, and Seydel (1929), labelled 1929. The orbit determined for LSS4300 must be regarded as somewhat provisional at this stage, because it is based on relatively few points ( 7 from our work and 8 from Jeffery et al. 1987).

The spectra were also used to examine the variability, and extent (with velocities ranging from -400 to $+550 \mathrm{~km} \mathrm{~s}^{-1}$ ), of the $\mathrm{H} \alpha$ profile, both as a function of orbital phase and over the time span of the study ( $\sim 4$ years). Figure 4 shows a sample of these spectra, stacked as a function of orbital phase. There is no dependence of the emission feature with phase, but the strength of the emission varies greatly over the period of the observational program. This was also noted by Greenstein in his series of papers.

There are still not sufficient data to enable a definitive model to be developed for this very unusual system, but there seems to be more than one mass loss process occurring in this binary system. A model developed by Nariai (1967) made some predictions about an anomalous high velocity $\mathrm{H} \alpha$ absorption line and some aspects of the emission, but the non-orbital related high velocity emission 


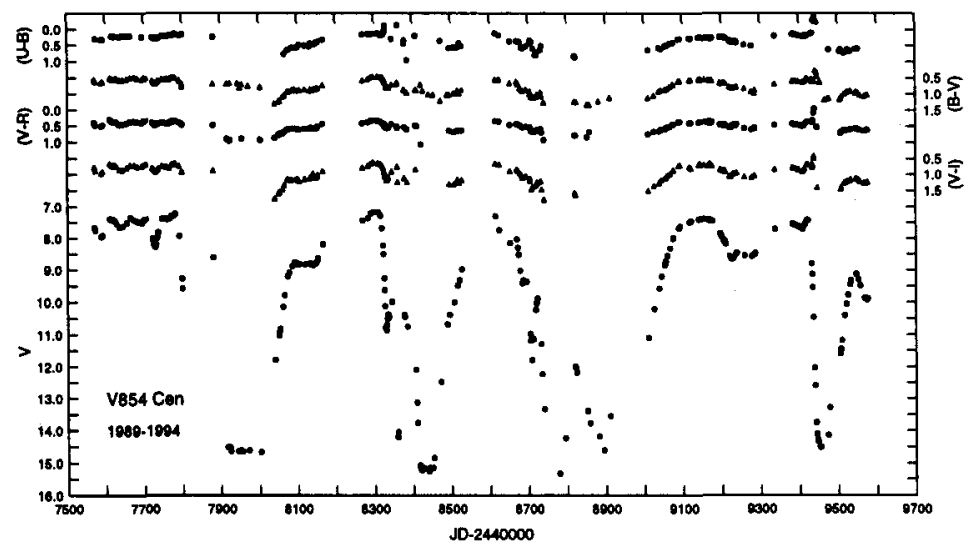

Figure 5. MJUO $V$ and colour photometry of V854 Cen.

must originate in a separate stream of material. A paper on these observations is in press (Frame et al. 1995).

\subsection{RCB stars}

Over the last 15 years there has been increased attention paid to this class of peculiar star. Their peculiar abundances (see Lambert \& Rao 1994) and anomalous variability (see Cottrell et al. 1990; Lawson et al. 1990), where they undergo large scale declines in brightness, have interested a wide range of photometrists, spectroscopists and modellers. There are also implications for stellar chromosphere studies, dust formation and evolution, and the later stages of stellar evolution.

MJUO photometry program At MJUO we have built up a database consisting of $\sim 9$ years of $(U) B V R I$ photometric data on up to 15 RCB stars. (A similar long-term program, on $\mathrm{R} \mathrm{CrB}$ itself, has been carried out by Fernie \& Seager (1994) using APT techniques.) These data have enabled detailed studies to be made of the pulsational characteristics of the stars (Lawson et al. 1990), with the more recent data containing photometry of many declines. Examples of the more recent data for the 'ultimate galactic roller coaster' star, V854 Cen is shown in Figure 5. Analysis of the extensive data set is in progress (Cottrell et al. 1995).

There have been a number of papers which have shown or endeavoured to make predictions about the time of decline (Dick \& Walker 1992) and whether there is any correlation between the decline onset time and the pulsations $(\mathrm{Pu}-$ gach 1977; Lawson et al. 1992). Although there are some indications, there is not a general trend.

As indicated in the introduction, the need to have magnitude and colour information available on these types of objects has been more than justified by the requests that we have had from other researchers. Generally they have obtained infrequent spectroscopic observations during the decline phase, and 
wish to know what the photometric state of the star was at that time. It cannot be stressed too strongly how vital that photometric information has become.

Spectroscopic program As indicated in the previous sections, there have been numerous studies of RCB stars both for abundance work in our Galaxy (see Lambert \& Rao 1994) and extragalactic systems (see Pollard et al. 1994), as well as to investigate the spectroscopic variations (at ultraviolet and optical wavelengths) during the pulsations at maximum light (see Clayton et al. 1994) and during various phases of the declines. Many more studies, particularly of the decline phase, are required to enable a consistent model of this phenomenon to be developed.

Other work and modelling There have been a number of new observational developments and some progress in the modelling of the decline events.

Recent discoveries of nebulae around RCB, hot RCB stars or related objects (see Pollacco et al. 1991; Pollacco \& Hill 1994) and 2 new RCB stars (Benson et al. 1994; Jurscik 1994) have rekindled the search for the remnants of mass loss or mass ejection processes from these stars and increased the possibility that with systematic search strategies there is some hope for an increase in the number of RCB or related stars. This can only help with various statistical studies about decline characteristics and provide further input for evolutionary modellers in terms of the expected lifetime for these stars.

There have been a number of groups working on the dust problem around RCB stars. It is now generally thought that the dust forms close to the photosphere (Clayton et al. 1992) and this group has worked on empirical models to show the general characteristics of this. Pugach and his co-workers (see Pugach \& Kovalchuk 1994) have developed a geometrical interpretation to the decline light curve, sometimes having a number of clouds which are simultaneously causing dimming of the RCB star. Some dust formation specialists (e.g., Goeres \& Sedlmayr 1992) have also been involved in some detailed modelling projects to form dust in the proximity of RCB stars. However, there are still serious problems in getting the dust to form sufficiently close (within $2 R_{*}$ ) of the stellar surface, a requirement of the more recent observational data.

\section{Cepheids}

The final area that I would like to address in this review of yellow supergiants is in Cepheid star research, far removed from the semi-regular or irregular stars already discussed. In particular, I wish to consider the spectroscopic characteristics of a number of these stars. This work began with the visit of George Wallerstein to Canterbury in 1988 and has continued since then with Michael Albrow and others.

\subsection{Previous work}

Wallerstein, Jacobsen and their various co-workers, as well as the CORAVEL consortium have provided much of the radial velocity work on Cepheids. (Apologies to other researchers who have been involved, but space precludes listing everbody!) Most of this work has centred on getting well-defined velocity curves 
to combine with various photometric data in order to use the Baade-Wesselink method to deduce radii and then distances, as well as tests for stellar evolutionary theories.

\subsection{MJUO and other work}

We were also involved in the quest for radial velocity curves (Wallerstein et al. 1992), but got 'side-tracked' by our series of observations which showed that there were small, but significant, asymmetries in the metallic spectral lines of all the Cepheids that we were investigating using MJUO facilities.

The analysis of these data involved the use of the line bisector method to determine the wavelength (and hence velocity) of a spectral line at a range of depths in the profile (Albrow \& Cottrell 1994). Different techniques have been adopted by others (Sasselov \& Lester 1990; Butler 1993) to make the appropriate measurements to quantify the line formation depth dependence of a spectral line.

\subsection{Modelling}

This detailed line profile analysis and line asymmetry has lead to further refinements in hydrodynamical models of Cepheids. In particular, there have been a number of studies which are seeking to provide full dynamical envelope and atmosphere models which will provide an opportunity to really understand the details of line formation processes in dynamic atmospheres.

Albrow \& Cottrell (1995a,b) have constructed hydrodynamic envelope models from which 'snap shot' dynamic atmospheres have been produced and synthetic line profiles have been calculated for comparison with the observational data. As a consequence of this, new projection factors have been calculated for use with Cepheid analyses and a new approach to the understanding of the formation of spectral lines in moving atmospheres has been developed using flux contribution functions.

Gillet and various co-workers (see Breitfellner \& Gillet 1993) have also been considering ways in which line profiles are formed. These studies have confirmed the need for a better understanding of the line formation processes in dynamic stellar atmospheres.

\section{Summary}

This paper has highlighted the importance of and need for continued long-term, contemporaneous photometric and spectroscopic work on a variety of variable yellow supergiant stars for a variety of astrophysical reasons.

Observers need to incorporate observations from a variety of other wavelengths in order to more completely define the parameters of a given system, e.g., binaries, circumstellar shells, chromospheres and dust formation.

There is also a need for more detailed hydrodynamical envelope and model atmosphere work to complement the available spectroscopic and photometric observations. This could lead to more complete models of a number of systems, in particular the semi-regular (e.g., the RV Tauri and RCB stars) and regular (e.g., Cepheids) variables. 
Acknowledgments. The invitation to present this paper would not have been possible without the involvement of my collaborators at Canterbury, who have helped me in this work over the last 10 years: Michael Albrow, Mike Clark, David Frame, Alan Gilmore, Pam Kilmartin, Warrick Lawson, Karen Pollard and the technical support available at the Observatory and University. I would also like to thank Karen Pollard and Michael Albrow for comments on the manuscript and Karen for generating Figures 3 and 4.

\section{References}

Aikawa, T. 1993, MNRAS, 262, 893

Albrow, M.D., \& Cottrell, P.L. 1994, MNRAS, 267, 548

Albrow, M.D., \& Cottrell, P.L. 1995a, these proceedings

Albrow, M.D., \& Cottrell, P.L. 1995b, MNRAS, in press

Benson, P.J., et al. 1994, AJ, 108, 247

Breitfellner, M.G., \& Gillet, D. 1993, A\&A, 277, 524

Buchler, J.R., \& Kovács, G. 1987, ApJ, 320, L57

Buchler, J.R., et al. 1995, Phys.Rev.Lett, 74, 842

Bulter, R.P. 1993, ApJ, 415, 323

Clayton, G.C., et al. 1992, ApJ, 397, 652

Clayton, G.C., et al. 1994, ApJ, 432, 785

Cottrell, P.L., Lawson, W.A., \& Buchhorn, M. 1990, MNRAS, 244, 149

Cottrell, P.L., et al. 1995, in preparation

Dick, J.S.B., \& Walker, H.J. 1992, A\&A, 252, 701

Dudley, R.E., \& Jeffrey, C.S. 1990, MNRAS, 247, 400

Dudley, R.E., \& Jeffrey, C.S. 1993, MNRAS, 262, 945

Fernie, J.D., \& Seager, S. 1994, PASP, 106, 1138

Fokin, A.B. 1994, A\&A, 292, 133

Frame, D.J., et al. 1995, in press

Gillet, D. 1992, A\&A, 259, 215

Giridhar, S., Rao, N.K., \& Lambert, D.L. 1994, preprint

Goeres, A., \& Sedlmayr, E. 1992, A\&A, 265, 216

Greenstein, J.L. 1950, ApJ, 111, 20

Jeffery, C.S., Drilling, J.S., \& Hill, P.W. 1987, MNRAS, 225, 1005

Jurscik, J. 1994, Acta Astron., 43, 353

Lambert, D.L. \& Rao, N.K. 1994, JA\&A, 15, 47

Lawson, W.A. et al. 1990, MNRAS, 247, 91

Lawson, W.A. et al. 1992, MNRAS, 256, 339

Lèbre, A., \& Gillet, D. 1991a, A\&A, 246, 490

Lèbre, A., \& Gillet, D. 1991b, A\&A, 251, 549

Luck, R.E., \& Bond, H.E. 1984, ApJ, 279, 729

Luck, R.E., \& Bond, H.E. 1989, ApJ, 342, 476 
Malcolm, G.J., \& Bell, S.A. 1986, MNRAS, 222, 543

Morrison, K. 1988, MNRAS, 233, 621

Nariai, K. 1967, PASJ, 19, 564

Percy, J.R. 1993, Ap\&SS, 210, 123

Pollacco, D.L. \& Hill, P.W. 1994, MNRAS, 267, 692

Pollacco, D.L., et al. 1991, MNRAS, 248, P1

Pollard, K.R., Cottrell, P.L., \& Lawson, W.A. 1994, MNRAS, 268, 544

Preston, G.W., et al. 1963, ApJ, 137, 401

Pugach, A.F. 1977, IBVS, 1277

Pugach, A.F., \& Kovalchuk, G.U. 1994, Astr Zh, 71, 250

Sasselov, D. 1993, unpublished preprint

Sasselov, D., \& Lester, J.B. 1990, ApJ, 362, 333

Seydel, F.L. 1929, PAAS, 6, 278

Shenton, M., et al. 1992, A\&A, 262, 138

Takeuti, M., \& Petersen, J.O. 1983, A\&A, 117, 352

Tuchman, Y., et al. 1993, A\&A, 271, 501

Wahlgren, G.M. 1992, ASP Conf. Series, 45, 270

Wallerstein, G., et al. 1992, MNRAS, 259, 474

Wallerstein, G., \& Elgar, S. 1992, Science, 256, 1531

Warner, B. 1986, in IAU Symp 118, 3

\section{Discussion}

Habing: This is a political statement in line with your opening remarks. There is a permanent danger that small telescopes will be shut down because large telescopes are so costly to build or to optimise. I think that a constant pressure should be exercised against this danger. Conferences like these and commissions of the IAU are the bodies from where we can exercise counterpressure.

Cottrell: I agree that we must exert pressure, but we need to ensure that excellent projects are proposed for these small telescopes and that specific objectives are set to answer specific astrophysical problems.

Percy: What fraction of the yellow supergiants are RV Tauri stars and what fraction are SRds?

Cottrell: I would estimate that a small fraction of yellow supergiants are RV Tauri stars and that a somewhat larger fraction are classified as of SRd type. However, the boundaries between the different types may be quite blurred and hence subdivision in this way may not be a perfectly satisfactory way of classifying semi-regular variables.

Percy: Could you comment on the rather frequent occurrence of RVb stars, with their interesting and puzzling obscurations?

Cottrell: The long period obscuration episodes in the RVb subclass of RV Tauri stars has been described by a number of models, although our new photometric 
and spectroscopic data indicate that the orbit is such that some type of active interaction phase occurs when the stars are at their closest approach and the RV Tauri star is closest to the observer (see Pollard et al., these proceedings).

Mattei: You may be interested in my poster in which I show 30-year light curve of $U$ Mon using the observations from the AAVSO International Database.

Cottrell: The record of the AAVSO offers key data for long-term study of many variables. However, there is also a need for photoelectric colours to enable other information to be inferred about these systems.

Mattei: There are two southern long period variables, $\mathrm{R}$ Cen and $\mathrm{R}$ Nor, that show RV Tauri (RVa) type variability, with deep and shallow alternating minima. Would you please comment if and how they may be related to the RV Tauri stars?

Cottrell: I would expect that these variables are related to the RV Tauri stars and that the alternating deep-shallow effect is some form of double mode resonance that may be ascribed to a much larger group of stars. With more extensive photometric and spectroscopic coverage we may be able to combine some of the wide variety of star classes into a more compact set.

Welch: Could you explain why the colour changes are different in nature from decline to decline in the RCB stars?

Cottrell: The different colour changes for different declines in the RCB stars are probably a combination of a number of effects, including the height of formation in the stellar atmosphere of the obscuring material, its growth rate and the level of excitation of the upper atmospheric (chromospheric) layers. This combination can consequently give very 'blue' declines when much of the photosphere but little of the chromosphere is initially obscured, to 'red' declines where either the chromosphere is obscured at the same rate as the photosphere or the chromosphere is in a quiescent state. The latter two situations would then not exhibit large numbers of emission lines in the spectra of the declines and hence would not have excessively blue colours.

Waelkens: Can you comment on the correlation between declines and pulsations in RCB stars? The absence of enhanced infrared emission during declines suggests to me that a decline is more a line-of-sight effect and that outbursts occur much more often on all sides of the star. It seems then hard to understand how the observed start of a decline correlates well with a pulsation phase, the more so that during most pulsation cycles no decline is observed.

Cottrell: There are at least two stars (V854 Cen and RY Sgr) in which there is evidence for a correlation between declines and pulsations (Lawson et al. 1992; Pugach 1977) and there is further evidence that the obscuring material must be forming close to the star (Clayton et al. 1992). I agree that the declines are a line-of-sight effect, where the obscuring cloud forms and blocks out successively more and more of the photosphere (and chromosphere). The onset of the decline could be considered to be a pulsation in which the atmospheric conditions (e.g., the temperature and pressure in the outer atmosphere) are such that grain formation can commence. The characteristics of the decline will then depend upon the geometry of the cloud with respect to the star and the observer and the rate of grain formation. 